\title{
Revista Mexicana de
}

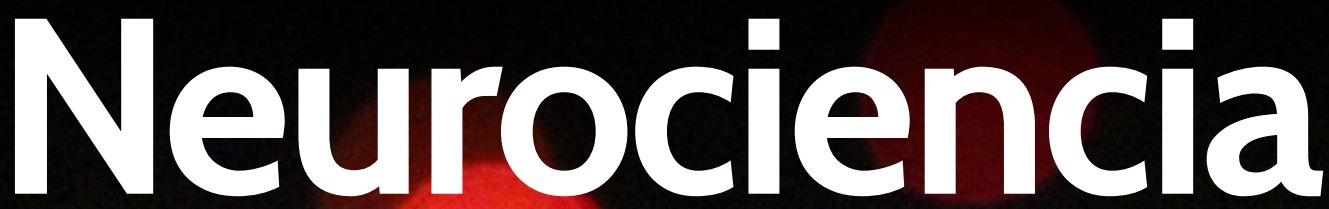

Publicación oficial de la Academia Mexicana de Neurología A.C.
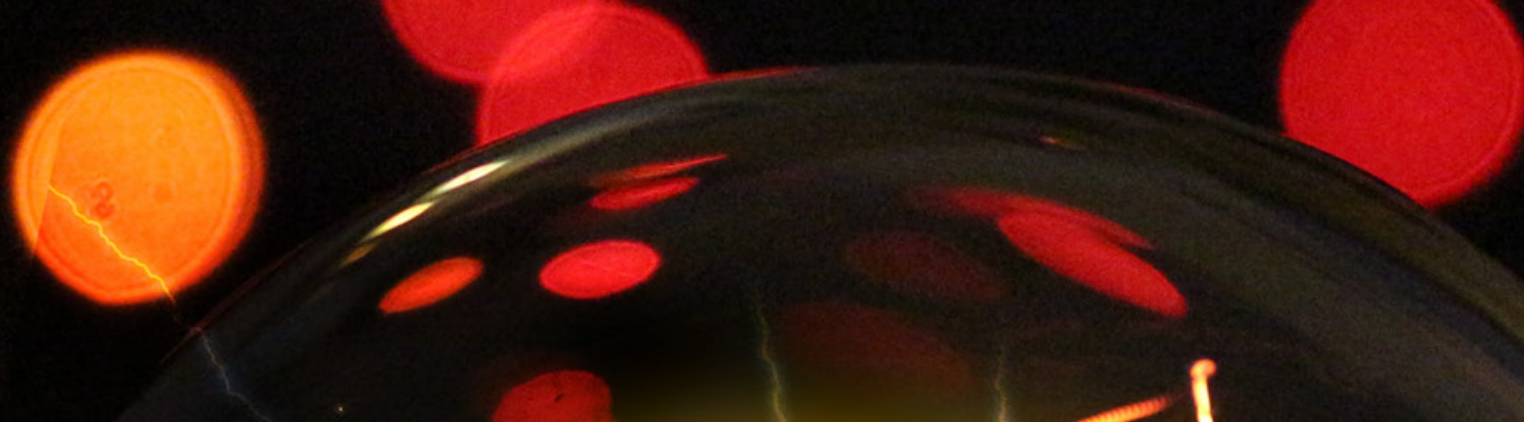


\section{Contribución original}

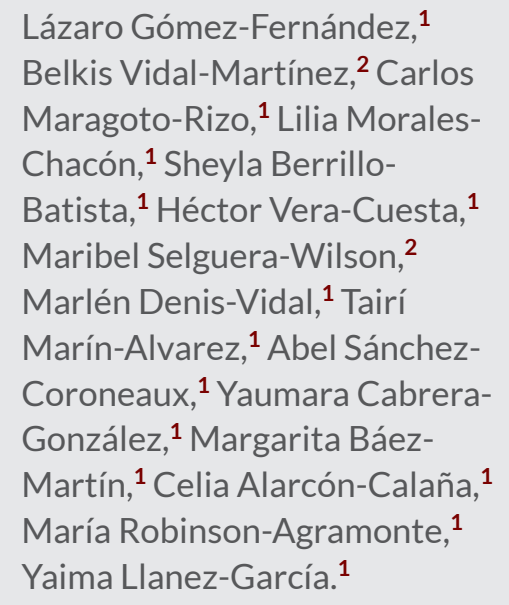

${ }^{1}$ Centro Internacional de Restauración Neurológica.

${ }^{2}$ Hospital Pediátrico Docente BorrásMarfán.

\section{Seguridad y efectividad de la Estimulación Cerebral No Invasiva en el Trastorno del Espectro del Autismo: Resultados de un estudio de prueba de concepto}

Safety and effectiveness of Non-Invasive Brain Stimulation in Autism Spectrum Disorder: Results from a proof of concept study

\section{Resumen}

Introducción. El Trastorno del Espectro del Autismo(TEA) es un desafío terapéutico para las neurociencias. Se han desarrollado diversas intervenciones farmacológicas y no farmacológicas con resultados beneficiosos; pero se requieren de más investigaciones para lograr mejores resultados clínicos. Existen evidencias sobre los beneficios potenciales de la Estimulación Cerebral No Invasiva(ECNI) en estos pacientes.

Objetivo. Evaluar la seguridad y la efectividad del uso de la ECNI en niños con TEA.

Métodos. Se realizó un estudio de prueba de concepto que incluyó 15 pacientes con diagnóstico de TEA según criterios del DSM-5, procedentes del Centro Internacional de Restauración Neurológica y del Hospital Pediátrico Borrás-Marfán(Habana, Cuba). Como criterio de inclusión se definió la no modificación del esquema terapéutico de los pacientes, y la existencia de estabilidad clínica 1 mes previo a la intervención. Se utilizaron dos métodos de ECNI: la estimulación magnética transcraneal repetitiva(rTMS) y la estimulación transcraneal con corriente directa(tDCS). Los pacientes hasta 10 años y 11 meses recibieron $\mathrm{tDCS}$ (cátodo $\mathrm{F3}$; ánodo brazo derecho); a partir de 11 años de edad la rTMS(1 Hz). La estimulación se focalizó sobre la corteza prefrontal dorsolateral del hemisferio izquierdo, con un total de 20 sesiones. Los pacientes fueron evaluados antes y una semana después de la intervención mediante la Escala de Evaluación de la Respuesta Terapéutica en el Autismo(ATEC), la Entrevista Diagnóstica para el Autismo(ADI-R) y la Lista de la Conducta Autista(ABC).

Resultados. En un paciente no fue posible iniciar el tratamiento 
por poca colaboración. Los restantes 14 completaron la intervención con pocos efectos adversos, básicamente molestias locales en el sitio estimulado. Se observó una disminución significativa en la puntuación de las escalas clínicas en la segunda evaluación (ADI-R: inicial $=52 \pm 9$,

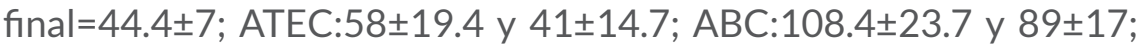
$\mathrm{p}<0.05)$; indicando una mejoría clínica grupal.

Conclusiones. La ECNI fue bien tolerada e indujo cambios conductuales en la muestra de pacientes en aspectos claves de la conducta autista.

\section{Palabras clave}

ECNI, rTMS, tDCS, Trastorno del Espectro del Autismo.

\author{
Correspondencia: \\ Dr. Lázaro Gómez Fernández \\ Laboratorio de EMG \& TMS. \\ Servicio de Neurofisiología Clínica y Unidad de Estimulación Cerebral No Invasiva. \\ Centro Internacional de Restauración Neurológica. \\ Calle 212, \%19 y 19. Atabey. Playa. Habana. Cuba \\ E.mail: lazarog@neuro.ciren.cu \\ Teléfono: 72736923
}




\section{Abstract}

Introduction. Autism Spectrum Disorder(ASD) still being a therapeutic challenge for neurosciences. Different pharmachological and non-pharmachological treatments have been developed inducing benefits in ASD patients in some degree, with cumulative effect; but more research is needed to improve clinical results. There are evidences about the potential positive effects of Non-Invasive Brain Stimulation(NIBS) in ASD patients.

Objective. To evaluate the safety and effectiveness of NIBS in patients with ASD.

Methods. We carried out a proof of concept study including 15 children with ASD according to DSM-5 diagnostic criteria. They proceeded from the ambulatory services of the International Center for Neurological Restoration and Borrás-Marfán Hospital(Havana, Cuba). Inclusion criteriaestablished that childrenshould had nochange in their therapeutic approach one month before the intervention; and an equivalent period of stabilization of their symptoms. Repetitive Transcranial Magnetic Stimulation(rTMS) and Transcranial Direct Current Stimulation(tDCS) were used as stimulation methods; tDCS(cathode F3, anode right arm) was used in children under 10 years and 11 month; and $1 \mathrm{~Hz}$ rTMS for children over 11 years. Stimulation was focalized over the left dorsolateral prefrontal cortex, and a total of 20 session were applied. Patients were evaluated before and one week after the intervention applying the Autism Treatment Evaluation Checklist(ATEC), the Autism Diagnostic Interview(ADI-R) and the Autism Behavior Checklist(ABC).

Results. Only in one patient it was not possible to start the treatment because of poor collaboration. The other 14 completed the 20 sessions with a few adverse effects, basically local pain at the stimulation site. A significant change in clinical scales was observed, with lower scores in the second evaluation, (ADI-R: initial= 52 \pm 9 , final=44.4 \pm 7 ; ATEC: $58 \pm 19.4$ and $41 \pm 14.7$; ABC: $108.4 \pm 23.7$ and $89 \pm 17$; $<0.05)$; indicating a clinical improvement in the group of patients.

\section{Keywords}

NIBS, rTMS, tDCS, Autism Spectrum Disorder.
Conclusions. NIBS was well tolerated and induced behavioral changes in our sample of patients in key aspects of autistic behavior. 


\section{Introducción}

El Trastorno del Espectro del Autismo(TEA) de acuerdo a los criterios actuales definidos en el DSM-5 incluye como su nombre lo indica un espectro de manifestaciones caracterizadas por déficits graves y alteraciones generalizadas en múltiples áreas del desarrollo. En estos pacientes existen alteraciones en la interacción social, trastornos de la comunicación y la presencia de comportamientos, intereses y actividades restringidas y estereotipadas. Bajo el término TEA en la actualidad se agrupan 4 diagnósticos diferentes que existían en la anterior clasificación del DSM-IV; pero en esencia se trata de un cuadro clínico cuyo diagnóstico en la mayor parte de los casos entraña un alto grado de discapacidad. ${ }^{1}$ Las cifras de prevalencia del TEA fluctúan entre diferentes países desde valores tan altos como las que describe en USA de 1 cada 68 niños de hasta 8 años, ${ }^{2}$ en Francia 0.36 casos por cada 100 niños, ${ }^{3}$ y en México 1 caso cada 115 niños. ${ }^{4}$ En general en países en vías de desarrollo son más escasos los datos epidemiológicos fidedignos en esta esfera.

Los avances en las neurociencias de los últimos años han permitido conocer más acerca de la fisiopatología del TEA, especialmente en cuanto a los patrones de conectividad cerebral que se alejan de lo descrito en sujetos normales. ${ }^{5}$ Como en otras enfermedades, desafortunadamente no existen grandes avances en su abordaje terapéutico; si bien es de destacar que el uso de diversos métodos de tratamiento fundamentalmente no farmacológicos resultan favorables en cuanto al control de la sintomatología autista, facilitando una mejor integración del paciente con su medio familiar y social. ${ }^{6}$ Desde el punto de vista farmacológico continúa sin existir un fármaco específico para el tratamiento del TEA, destacándose a nivel internacional el uso de la risperidona, el aripiprazol, la carbamazepina, ácido valproico y el metilfenidato entre otros, según los síntomas que predominen y la comorbilidad con otras patologías. ${ }^{7,8}$ Todas las terapéuticas descritas en la literatura benefician a los pacientes con TEA, a las que se le suma también la Medicina Tradicional o Alternativa; pero por consenso se considera que se requieren de más investigaciones científicas para definir mejores estrategias terapéuticas que permitan alcanzar resultados clínicos superiores. ${ }^{9}$

Bailey y colaboradores fueron de los primeros en apuntar hacia la corteza cerebral como elemento clave en la fisiopatología del TEA $;{ }^{10}$ posteriormente Casanova y colaboradores llamaron la atención en una serie de investigaciones sobre existencia de anomalías en la organización minicolumnar de la corteza cerebral, entre ellas la existencia de neuronas más pequeñas, con incremento en su número y densidad por unidad de área, heterotopias periventriculares y displasias corticales; además de una reducción significativa del neurópilo, que se traduce en una disfunción inhibitoria intracortical; aspecto que posiblemente contribuya de forma importante a gran parte de la sintomatología autista. ${ }^{11,12}$

En los últimos años se han desarrollado una gran cantidad de investigaciones utilizando métodos de estimulación cerebral no invasiva(ECNI) como la estimulación magnética transcraneal repetitiva(rTMS) y la estimulación transcraneal con corriente directa(tDCS). Estos métodos dependiendo del protocolo específico que se utilice pueden tener un efecto potenciador de la inhibición cortical o de incremento de la excitabilidad en los circuitos sobre los que se incide. ${ }^{13,14}$ Tanto la rTMS como la tDCS son métodos que se utilizan ampliamente en la actualidad para el control sintomático de diversas enfermedades, pero con diferencias en cuanto a los niveles de evidencias científicas. ${ }^{15,16}$

Se han publicado varios artículos que aportan evidencias sobre el efecto favorable que pueden tener estos métodos en cuanto al control de la conducta autista, incluso con cambios en la expresión de algunas respuestas electrofisiológicas; aunque en su mayoría con la aplicación de un número bajo se sesiones como para esperar un efecto clínico significativo y duradero. ${ }^{17-19}$ Por otro lado no existen datos que permitan diferenciar la eficacia de larTMS y la tDCS como neuromoduladores, aspecto que reviste gran 
importancia como guía de selección de uno u otro método para uso terapéutico.

En el presente trabajo se parte de la hipótesis de que el uso de la ECNI puede tener un efecto adyuvante en el tratamiento de los niños con TEA, y nos planteamos como objetivos principales demostrar la seguridad del uso de la rTMS y la tDCS en este caso particular, así como obtener evidencias de tipo prueba de concepto sobre el efecto de ambos métodos para el mejor control de la sintomatología clínica.

\section{Métodos}

Se incluyeron en este estudio un total de 15 niños con diagnóstico de TEA, clasificados como moderados o ligeros; siguiendo los criterios del DSM-5 y partiendo de la coincidencia de la impresión diagnóstica del Especialista en Neuropediatría y Psiquiatría Infanto-Juvenil(13 varones y 2 hembras; edades entre 5 y 13 años). Los pacientes procedieron de los servicios de atención ambulatoria del Hospital Pediátrico Docente Borrás-Marfán y del Centro Internacional de Restauración Neurológica, atendidos en el período de Noviembre 2015-Octubre 2016.

Como criterios de inclusión se consideró la concordancia en la impresión diagnóstica por parte del Especialista en Neuropediatría y en Psiquiatría Infanto-Juvenil; el mantenimiento del mismo esquema terapéutico(farmacológico y psicopedagógico) durante el último mes previo del inicio de la intervención, y la estabilidad de la sintomatología en igual período de tiempo. El diagnóstico clínico fue confirmado mediante la aplicación de la Escala de Valoración del Autismo Infantil(CARS) ${ }^{20}$ y la Entrevista Diagnóstica para el Autismo(algoritmo diagnóstico), Edición Revisada(ADI-R) que fueron aplicadas por 2 Especialistas en Psiquiatría Infantil calificados para su uso. ${ }^{21}$

Fueron excluidos de la selección inicial aquellos pacientes en los que existía comorbilidad con la epilepsia, o en los que se efectuaron modificaciones en la farmacoterapia(en dosis o en tipo de medicamento) en el último mes previo su reclutamiento para esta investigación. En ningún caso se interrumpió el esquema terapéutico de base que tenía cada paciente, solo se le añadió el protocolo de estimulación propuesto según su edad.

\section{Diseño experimental}

Los pacientes fueron reclutados mediante inclusión continua, según se fueron presentando en sus correspondientes servicios de atención; se les explicó a los padres las características del estudio y se les asignó atendiendo a su edad al grupo de tratamiento con tDCS(5 años- 10 años y 11 meses) o rTMS(11 años en adelante). Esta diferenciación se estableció por la mejor colaboración en general de los niños mayores de 10 años para la realización de los diferentes procederes evaluativos, y el requerimiento técnico más exigente para la aplicación de la rTMS. La tDCS se aplicó con un equipo Neuroconn(GE), y se colocó el cátodo en posición de F3(sistema 10/20), como guía de proyección de la corteza prefrontal dorsolateral del hemisferio izquierdo, y el ánodo sobre el tercio superior del brazo derecho. Cada sesión tuvo una duración de 20 minutos y se estimuló con $1 \mathrm{~mA}$ de intensidad. En el caso de la rTMS se aplicaron en cada sesión un total de 1500 pulsos, con frecuencia de $1 \mathrm{~Hz}$ y una intensidad del 90\% del valor del umbral motor(MagStim, UK), colocando el centro de una bobina refrigerada en forma de 8 sobre la misma posición. Durante las sesiones de estimulación los pacientes se mantuvieron sentados cómodamente, se les proyectaron dibujos animados de su preferencia en una pantalla de televisión, y en algunos casos también se les permitió manipular juguetes didácticos y tabletas con juegos de entretenimiento, para facilitar su cooperación. Todos recibieron 20 sesiones de tratamiento en total.

Los pacientes fueron evaluados en dos ocasiones antes de iniciar la intervención aplicando el ADI$\mathrm{R}$ (algoritmo para condición actual); ${ }^{21}$ la Lista 
de la Conducta Autista(ABC $)^{22}$ y la Lista para la Evaluación de la Respuesta Terapéutica en el Autismo(ATEC). ${ }^{23}$ Estas evaluaciones fueron nuevamente realizadas una semana después de completar las 20 sesiones de tratamiento. En ningún caso los evaluadores participaron directamente en la aplicación de las sesiones de estimulación.

Adicionalmente se les realizó un electroencefalograma(EEG) en estado de vigilia antes de iniciar el tratamiento y una semana después de haber culminado este, con el objetivo de corroborar si se identificaban modificaciones en los patrones de conectividad cerebral. Se utilizó un montaje de 19 electrodos activos de Ag-Cl sobre el cuero cabelludo(Fp1, Fp2, F7, F8, F3, F4, C3, C4, T5, T6, T3, T4, P3, P4, O1, O2, Fz, $\mathrm{Cz}, \mathrm{Pz}$ ), según el sistema internacional 10/20, con referencias a nivel de $\mathrm{Cz}$ y $\mathrm{Pz}$. La impedancia se mantuvo por debajo de $5 \mathrm{KOhm}$. Para dicho análisis en el registro de cada paciente se seleccionaron 38 ventanas libres de artefactos de los registros electroencefalográficos en estado de vigilia con ojos abiertos. La conectividad funcional se analizó a partir del cálculo de la matriz de sincronización espacial (en inglés synchronization likelihood) entre los electrodos. ${ }^{24,25}$ Se determinó para las cinco bandas de frecuencia Alfa(8-12.9 Hz), Beta(13-29.9 Hz), Theta(4-7.9 Hz), Delta(1-3.9 Hz) y Gamma(solo en el rango de 30-35 Hz). 26 Todo el procesamiento se realizó mediante algoritmos implementados sobre el programa MATLAB R2008b, y se compararon las matrices de datos de cada paciente antes de la intervención con los registrados posterior a la intervención.

\section{Efectos adversos}

Durante las sesiones de estimulación se mantuvo una estrecha observación de los niños, buscando signos que denotaran molestias locales, o cualquier manifestación de hiperexcitabilidad cortical anormal inducida por la intervención. Al día siguiente se interrogó a los padres sobre cualquier cambio conductual que hubiesen notado en el niño, especialmente en las primeras horas transcurridas al terminar las sesiones.

\section{Ética médica}

El protocolo fue aprobado por el Consejo Científico y el Comité de Ética Institucional del Centro Internacional de Restauración Neurológica. Se contó con el consentimiento informado por escrito de los padres, quienes recibieron una amplia explicación oral y por escrito sobre las características de los procedimientos. Se cumplieron con los lineamientos éticos para la investigación en humanos recogidos en el Código de Ética de la Asociación Médica Mundial (Declaración de Helsinki) para experimentos que involucran humanos. ${ }^{27}$

\section{Análisis estadístico}

Se realizó un análisis estadístico no paramétrico utilizando el test para series pares de Wilcoxon, para la comparación del comportamiento de las escalas en los dos momentos evaluativos; y $U$ de Mann-Whitney para definir si existían diferencias en cuanto a la respuesta terapéutica entre grupos con diferentes intervenciones. En ambos casos se consideró un $\alpha=0.05$ (Statistica 7.0, Stat Soft Inc. 2004). En el caso del análisis de conectividad basado en el EEG, también se consideró un $\alpha=0.05$ para las comparaciones entre las matrices de datos extraídas de los dos momentos evaluativos, con el uso de una t de Student.

\section{Resultados}

\section{Tolerancia y efectos adversos}

La distribución final de la muestra según el método de tratamiento incluyó un total de 9 niños menores de 11 años que recibieron tDCS y 6 niños mayores de 11 años que recibieron rTMS; solo 2 de ellos fueron del sexo femenino. La mayoría de los pacientes mostró buena tolerancia con ambos procedimientos, y solo en un niño de 8 años(tDCS) no se logró realizar la terapia por 
falta de colaboración después de varios intentos de colocar los electrodos para la estimulación en días no consecutivos. En los restantes pacientes fue posible comenzar el tratamiento el mismo día en que se propuso su inicio y completar el mismo sin interrupción. El efecto adverso más frecuente referido o detectado a partir de la conducta de los niños fue la existencia de molestias locales en el sitio de la estimulación( $\approx 50 \%$ ), que en todos los casos fueron ligeras, sin requerir ninguna intervención farmacológica o de otro tipo(Tabla 1). La somnolencia ocupó el segundo lugar, que en realidad atendiendo a la descripción de los padres se expresó más bien en que el niño comenzó a dormir en la noche 1 o 2 horas más temprano de lo habitual, y en general con un sueño más tranquilo y regular, aunque este no fue un aspecto que se evaluó en profundidad.

\section{Efectos de la intervención}

En todos los casos de forma individual los padres identificaron cambios conductuales descritos cualitativamente de forma espontánea y también transmitidos indirectamente por sus maestros y demás familiares, que con frecuencia no estaban al tanto de que el niño estaba recibiendo una intervención experimental.Sedestacóentreestos cambios los relacionados fundamentalmente con la mejoría en su socialización y en la comunicación; adicionalmente disminución en la variedad y cantidad de estereotipias, desde la misma primera semana posterior a finalizar la intervención.

Las evaluaciones inicial y post-intervención se desarrollaron manteniendo exactamente las mismas condiciones de escenario, variedad de juguetes e interacciones. Los evaluadores de forma similar describieron cambios conductuales cualitativos y cuantitativos en cuanto a las interacciones del niño durante la entrevista, las característicasdeljuegoyel gradodehiperactividad. Estos cambios se reflejaron en los resultados del análisis estadístico de las puntuaciones de las escalas clínicas evaluadas (Figura 1).

La reducción en la puntuación de las escalas fue del $15 \%$ con respecto a la puntuación inicial de forma grupal una semana después de completar la intervención. Por otro lado no se

Tabla 1. Características generales y efectos adversos observados en la muestra estudiada. TDAH: Trastorno por Déficit de Atención e Hiperactividad. CBZ: carbamazepina; RPD: risperidona

\begin{tabular}{|c|c|c|c|c|c|c|c|c|c|c|}
\hline Paciente & EDAD & Sexo & ECNI & Comorbilidad & $\begin{array}{c}\text { Tratamiento } \\
\text { farmacológico }\end{array}$ & $\begin{array}{l}\text { Grado de } \\
\text { severidad }\end{array}$ & $\begin{array}{l}\text { Completó el } \\
\text { tratamiento }\end{array}$ & $\begin{array}{c}\text { Molestias } \\
\text { locales }\end{array}$ & Cefalea & Somnolencia \\
\hline 1 & 5 & $M$ & tDCS & - & CBZ & Ligero & Sí & Sí & - & - \\
\hline 2 & 5 & $M$ & tDCS & - & CBZ, RPD & Ligero & Sí & - & - & - \\
\hline 3 & 8 & M & tDCS & - & CBZ & Moderado & No & - & - & - \\
\hline 4 & 10 & $M$ & tDCS & - & CBZ, RPD & Moderado & Sí & - & - & - \\
\hline 5 & 13 & $M$ & rTMS & - & - & Moderado & Sí & - & - & Sí \\
\hline 6 & 13 & $M$ & rTMS & - & CBZ, RPD & Moderado & Sí & - & - & - \\
\hline 7 & 13 & $M$ & rTMS & - & CBZ & Ligero & Sí & - & - & Sí \\
\hline 8 & 7 & $\mathrm{~F}$ & tDCS & - & : & Ligero & Sí & Sí & - & Sí \\
\hline 9 & 13 & M & rTMS & - & CBZ & Moderado & Sí & - & Sí & Sí \\
\hline 10 & 9 & $M$ & tDCS & - & RPD & Ligero & Sí & Sí & - & - \\
\hline 11 & 10 & $\mathrm{~F}$ & tDCS & - & CBZ & Ligero & Sí & Sí & - & - \\
\hline 12 & 8 & $M$ & tDCS & - & - & Ligero & Sí & Sí & - & - \\
\hline 13 & 10 & $M$ & tDCS & - & CBZ & Moderado & Sí & Sí & - & - \\
\hline 14 & 7 & $M$ & tDCS & TDAH & CBZ & Moderado & Sí & Sí & - & - \\
\hline 15 & 11 & $M$ & rTMS & - & CBZ & Ligero & Sí & - & - & Sì \\
\hline
\end{tabular}


identificaron diferencias en cuanto a la magnitud de las modificaciones observadas en las escalas en relación al método utilizado(tDCS vs rTMS), aún sin considerar la diferencias en el promedio de edad entre los grupos que recibieron las dos intervenciones $(\mathrm{tDCS}=8.7$ años; $\mathrm{rTMS}=12.6$ años), mostrando una eficacia equivalente ambos métodos como moduladores de la conducta autista(Figura 2).

Modificaciones en la conectividad cerebral basadas en el análisis de la actividad de base del electroencefalograma.
En análisis de conectividad basado en el EEG mostró cambios significativos posterior a la intervención, con un incremento en la conectividad funcional en las frecuencias alfa, beta y gamma; especialmente en esta última( $p<0.05)$. El mismo análisis para las frecuencias más lentas $(\theta$ y $\delta)$ tuvo un comportamiento contrario con disminución de la conectividad especialmente en regiones anteriores $(p<0.05)$. La distribución topográfica de este aumento en conectividad funcional para las frecuencias más rápidas tuvo un carácter más difuso, incluyendo todas las regiones, con una distribución más amplia en el caso de la gamma(Figura 3).

Figura 1. Comportamiento de las puntuaciones en las escalas clínicas con la intervención (* $p<0.05)$.

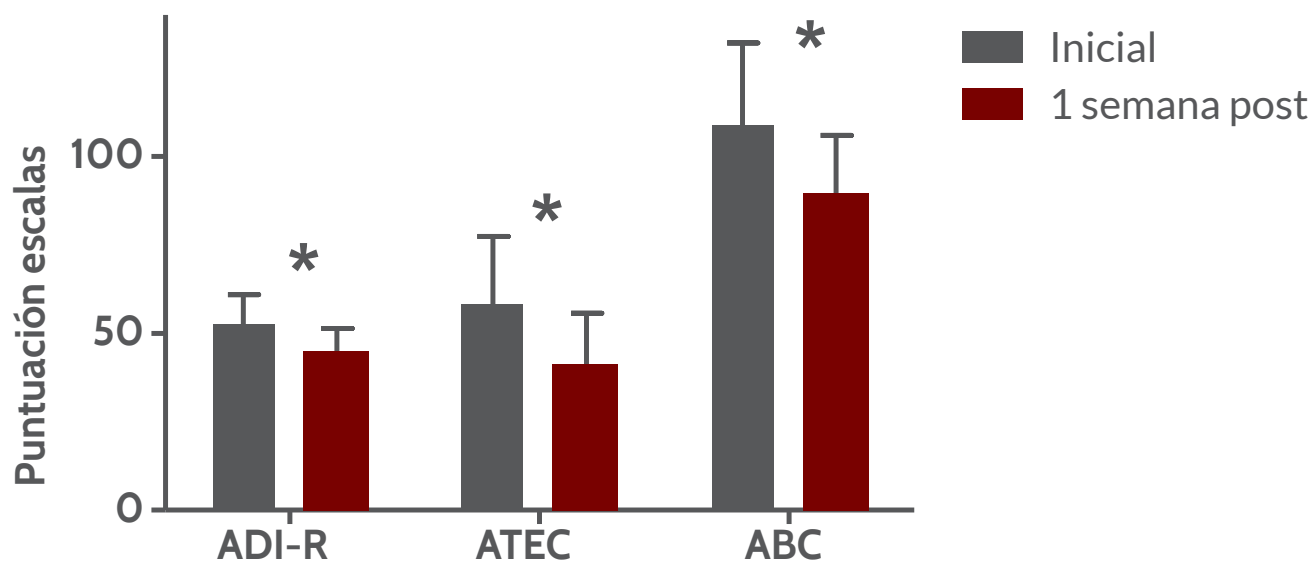

Figura 2. Diferencias entre las puntuaciones de las escalas clínicas antes y 1 semana después de completar el tratamiento con el uso de la tDCS y la rTMS ( $p>0.05$ en las 3 escalas).

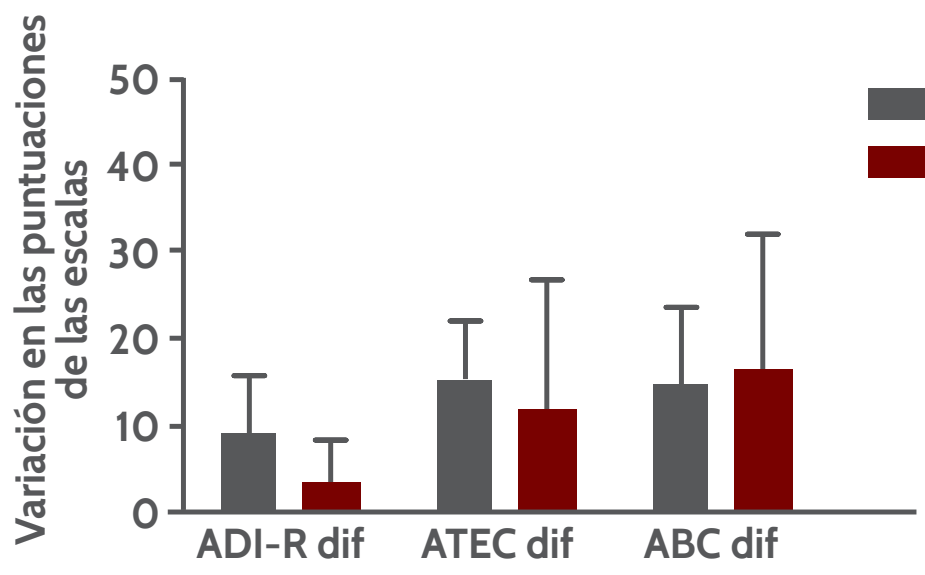


Figura 2. Incremento en la conectividad funcional entre los puntos seleccionados para las frecuencias analizadas $>8 \mathrm{~Hz}$ ( $\alpha, \beta$ y y (se representan solamente los cambios significativos, $p<0.05)$.

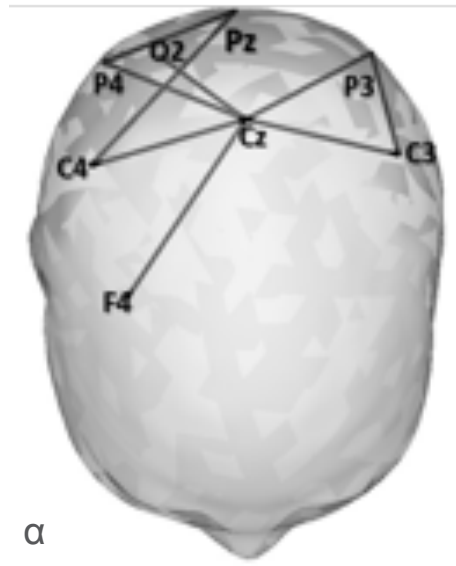

\section{Discusión}

La aplicación de los métodos de ECNI en población pediátrica ha estado limitada en años previos ante la falta de datos sobre su seguridad y efectos adversos potenciales; a tal punto que aún en la literatura de referencia como contraindicación formal para la aplicación de la rTMS se encuentran los niños menores de 18 años. La ausencia de evidencias sobre su seguridad no ha significado en ningún momento que existan evidencias que contraindiquen su uso en menores de 18 años. Afortunadamente solo ha sido cuestión de tiempo demostrar que tanto la aplicación de la rTMS como la tDCS son métodos seguros, tolerables, y que se asocian con muy pocos efectos adversos, tanto en sujetos sanos como en pacientes..$^{28,29}$

Los efectos adversos más frecuentes que se describen con ambos métodos consisten en la presencia de molestias locales, observación que compartimos con los resultados de este trabajo..$^{30,31}$ En segundo lugar, los cambios observados por los padres en cuanto al sueño, señalados como somnolencia, se limitan en todos los casos a que el niño se acuesta más temprano de lo acostumbrado, y duerme en la mayor parte de la noche sin interrupciones; aún en aquellos pacientes que dormían poco o tenían un sueño muy intranquilo con despertares frecuentes. Esta descripción la interpretamos como una mejoría en la estructura y calidad del sueño, sin que este haya sido un objetivo preconcebido como parte de la investigación. Evidentemente se trata de un efecto colateral beneficioso en todos los casos en los que se reportó, y es un efecto conocido, pero menos reportado en la literatura. ${ }^{32}$ En ningún caso se presentaron convulsiones ni otros fenómenos de hiperexcitabilidad cortical anormal.

Por supuesto que el resultado más interesante en esta investigación radicó en la descripción de una mejoría significativa en la sintomatología autista en todos los niños que recibieron la intervención, logrando una reducción adicional a la obtenida con el tratamiento de base de un 15\% en la puntuación de las escalas clínicas utilizadas. Esto coincidió cualitativamente con la impresión clínica global de los evaluadores, que en su mayoría eran además los especialistas que seguían a estos pacientes desde su diagnóstico inicial. Como aspecto destacado tanto padres como evaluadores señalaron una mejoría muy significativa en la socialización(basados en elementos cualitativos subjetivos), aunque desde el punto de vista de las escalas clínicas el efecto fue más global; y en segundo lugar mejoraron todos los aspectos del lenguaje. A diferencia de investigaciones publicadas por otros grupos, en 
nuestro caso se utilizó un número total de sesiones mucho mayor de la que han descrito hasta el momento en pacientes con TEA, ${ }^{33,34}$ y el resultado clínico nos parece muy consistente al contrastar el comportamiento de las escalas utilizadas y el cambio conductual cualitativo en los pacientes.

En esta investigación no se identificaron diferencias significativas en cuanto al efecto modulatorio entre larTMSylatDCSenlaexpresióndelasintomatología autista; en ambos grupos los valores se movieron después del tratamiento en un rango similar, y a pesar de que la edad promedio de los grupos fue diferente por decisión propia de los investigadores. Tal efecto no parece estar relacionado con la edad de los pacientes. Este es un elemento que no se ha abordado en la literatura y puede ser un punto de partida interesante pues contrasta el uso de un método más costoso y exigente desde el punto de vista técnico(rTMS) con otro más barato, técnicamente menos exigente y con resultados similares, de tal forma que la relación costo beneficio puede estar en favor del uso extendido de la tDCS. Por supuesto que la rTMS es en la actualidad el método de ECNI que cuenta con un mayor número de evidencias científicas, esta es solo una hipótesis que requiere de más investigaciones para corroborar su veracidad. ${ }^{35-37}$ Particularmente en el caso del TEA la ECNI no se puede recomendar como alternativa terapéutica válida por falta de evidencias científicas suficientes, pero existen varios indicios que permiten apostar porque en un futuro próximo sí lo será. Aunque también llama la atención la heterogeneidad en cuanto a métodos se refiere, al analizar los resultados de pequeños ensayos clínicos publicados con buenos resultados aparentes y el uso de polaridades de estimulación invertidas para el caso de la tDCS. ${ }^{34,38}$

Estainvestigacióntienelalimitantedequenocuenta con un grupo control y/o placebo, con lo cual no se descarta completamente el factor subjetivo tanto en pacientes, familiares como entre los evaluadores; pero partimos de un diseño experimental en el que se controló el uso concomitante de otras terapéuticas; exigiendo además que el paciente transitara por un período de al menos 1 mes de estabilidad en su esquema terapéutico y también de estabilidad en su sintomatología. De esta forma e independientemente de que no existiera grupo control, siendo los propios pacientes que reciben la intervención sus propios controles, existe una alta probabilidad de los cambios observados sean secundarios a la intervención y no a otra causa. Tanto el incremento de la muestra como un diseño diferente incluyendo un grupo control con el uso de placebo resultan claves para llegar a conclusiones sólidas en cuanto al efecto de estas intervenciones.

Considerando los elementos que se han descrito sobre a fisiopatología del TEA, ${ }^{12,39}$ que de acuerdo a la teoría que seguimos en este trabajo parte de una disfunción inhibitoria intracortical que motiva entre otras cosas, las alteraciones en la conectividad funcional entre áreas cerebrales..$^{40} \mathrm{El}$ efecto beneficioso puede estar asociado al menos en parte al incremento de la conectividad funcional entre áreas corticales dependiente de la actividad de las frecuencias en el rango de la actividad $\alpha, \beta$ y $y$. Particularmente en cuanto a esta última banda de frecuencia, en el presente trabajo solo se analizó el componente y más bajo $(30-35 \mathrm{~Hz})$ en estado de vigilia, en reposo con ojos abiertos. Se sabe que el poder espectral de la actividad ? es dependiente del estado funcional del cerebro; y que su incremento se asocia generalmente al proceso de atención en sujetos normales, mostrando alto grado de coherencia interhemisférica. Al parecer en pacientes con TEA aunque la mayor parte de los estudios describen el incremento de la energía espectral de esta banda de frecuencias, se diferencia de los registros normales precisamente en su grado de coherencia. En el TEA se ha descrito que el incremento en la conectividad de la banda $y$ especialmente en regiones posteriores se asocia con un nivel de funcionamiento más elevado. ${ }^{41} \mathrm{De}$ cualquier manera parece existir un vínculo entre la actividad $y$ y el TEA, en nuestro caso, el incremento de conectividad funcional dependiente de la actividad gamma, se asoció a una mejoría clínica, sin que de momento podamos decir mucho más al respecto, pero evidentemente es un aspecto que merece más atención, quedando pendiente la definición del tipo de relación existente entre dicho cambio funcional y el efecto terapéutico de la ECNI. 


\section{Conclusiones}

El uso de la Estimulación Cerebral No Invasiva es tolerable en niños con Trastorno del Espectro del Autismo e induce cambios significativos en aspectos claves de la conducta autista, con incremento en la conectividad funcional entre áreas corticales dependiendo fundamentalmente de las frecuencias más rápidas.

\section{Conflicto de intereses}

Los autores declaran que en este estudio no existen conflictos de interés relevantes.

\section{Fuentes de financiamiento}

No existió una fuente de financiamiento particular para este informe científico. 


\section{Referencias}

1. American Psychiatric Association. Neurodevelopmental Disorders. In: American Psychiatric Association, ed. Diagnostic and Statistical Manual of Mental Disorders, Fifth Edition ed. Washington DC: American Psychiatric Publishing. 2013:31-86.

2. Christensen D, Van Naarden BK, Doernberg NS, et al. Prevalence of cerebral palsy, co-occurring autism spectrum disorders, and motor functioning - Autism and Developmental Disabilities Monitoring Network, USA, 2008. Dev Med Child Neurol. 2014 Jan;56:59-65.

3. van Bakel MM, Delobel-Ayoub M, Cans C, et al. Low but increasing prevalence of autism spectrum disorders in a French area from register-based data. J Autism Dev Disord. 2015 Oct;45:3255-3261.

4. Fombonne E, Marcin C, Manero AC, et al. Prevalence of Autism Spectrum Disorders in Guanajuato, Mexico: The Leon survey. J Autism Dev Disord. 2016 May;46:1669-1685.

5. Rane P, Cochran D, Hodge SM, Haselgrove C, Kennedy DN, Frazier JA. Connectivity in Autism: A Review of MRI Connectivity Studies. Harv Rev Psychiatry. 2015 Jul;23:223-244.

6. Mukherjee S, Rupani K, Dave M, Subramanyam A, Shah H, Kamath R. Evaluation of effectiveness of integrated intervention in autistic children. Indian J Pediatr. 2014 Apr;81:339-345.

7. Jobski K, Hofer J, Hoffmann F, Bachmann C. Use of psychotropic drugs in patients with autism spectrum disorders: a systematic review. Acta Psychiatr Scand. 2016 Sep 13;10.

8. Ruggieri VL, Arberas CL. [Therapeutic approaches in autism spectrum disorders]. Rev Neurol. 2015 Feb 25;60 Suppl 1:S45-9::S45-S49.

9. Brondino N, Fusar-Poli L, Rocchetti M, Provenzani U, Barale F, Politi P. Complementary and Alternative Therapies for Autism Spectrum Disorder. Evid Based Complement Alternat Med. 2015;2015:258589. doi: 10.1155/2015/258589. Epub;\%2015 May 7.:258589.

10. Bailey A, Luthert P, Dean A, et al. A clinicopathological study of autism. Brain. 1998 May;121:889905.

11. Casanova MF, El-Baz AS, Kamat SS, et al. Focal cortical dysplasias in autism spectrum disorders. Acta Neuropathol Commun. 2013 Oct 11;1:67. doi: 10.1186/2051-5960-1-67.:67-1.

12. Frye RE, Casanova MF, Fatemi SH, et al. Neuropathological Mechanisms of Seizures in Autism Spectrum Disorder. Front Neurosci. 2016 May 10;10:192. doi: 10.3389/fnins.2016.00192. eCollection;\%2016:192.

13. Muller-Dahlhaus F, Vlachos A. Unraveling the cellular and molecular mechanisms of repetitive magnetic stimulation. Front Mol Neurosci. 2013 Dec 17;6:50. doi: 10.3389/fnmol.2013.00050. eCollection;\%2013.:50.

14. Bikson M, Name A, Rahman A. Origins of specificity during tDCS: anatomical, activity-selective, and input-bias mechanisms. Front Hum Neurosci. 2013 Oct 21;7:688. doi: 10.3389/fnhum.2013.00688. eCollection;\%2013.:688.

15. Berlim MT, Van den Eynde F, Daskalakis ZJ. Efficacy and acceptability of high frequency repetitive transcranial magnetic stimulation (rTMS) versus electroconvulsive therapy (ECT) for major depression: a systematic review and meta-analysis of randomized trials. Depress Anxiety. 2013 Jul;30:614-623.

16. Lefaucheur JP, Antal A, Ahdab R, et al. The use of repetitive transcranial magnetic stimulation (rTMS) and transcranial direct current stimulation (tDCS) to relieve pain. Brain Stimul. 2008 Oct;1:337-344.

17. Casanova MF, Baruth JM, El-Baz A, Tasman A, Sears L, Sokhadze E. Repetitive Transcranial Magnetic Stimulation (rTMS) Modulates Event-Related Potential (ERP) Indices of Attention in Autism. Transl Neurosci. 2012 Jun 1;3:170-180.

18. Enticott PG, Rinehart NJ, Tonge BJ, Bradshaw JL, Fitzgerald PB. Repetitive transcranial magnetic stimulation (rTMS) improves movement-related cortical potentials in autism spectrum disorders. Brain Stimul. 2012 Jan;5:30-37.

19. Sokhadze EM, Baruth JM, Sears L, Sokhadze GE, El-Baz AS, Casanova MF. Prefrontal neuromodulation using rTMS improves error monitoring and correction function in autism. Appl Psychophysiol Biofeedback. 2012 Jun;37:91-102.

20. Schopler E, Reichler RJ, DeVellis RF, Daly K. Toward objective classification of childhood autism: Childhood Autism Rating Scale (CARS). J Autism Dev Disord. 1980 Mar;10:91-103.

21. Lord C, Rutter M, Le CA. Autism Diagnostic Interview-Revised: a revised version of a diagnostic interview for caregivers of individuals with possible pervasive developmental disorders. J Autism Dev Disord. 1994 Oct;24:659-685. 
22. Volkmar FR, Cicchetti DV, Dykens E, Sparrow SS, Leckman JF, Cohen DJ. An evaluation of the Autism Behavior Checklist. J Autism Dev Disord. 1988 Mar;18:81-97.

23. Al Backer NB. Correlation between Autism Treatment Evaluation Checklist (ATEC) and Childhood Autism Rating Scale (CARS) in the evaluation of autism spectrum disorder. Sudan J Paediatr. 2016;16:17-22.

24. Niso G, Pereda E, Gutiérrez R, Bajo R, Maestrú F, et al. HERMES: towards an integrated toolbox to characterize functional and effective brain connectivity. Neuroinformatics. 2013;11:405-34.

25. Stam C. Synchronization likelihood: an unbiased measure of generalized synchronization in multivariate data sets. Physica D. 2002;163:236-241.

26. Nierdermeyer E. The Normal EEG of the waking adult. In: Niedermeyer E and Lopez da Silva, eds. Electroencephalography. Basic principles, clinical applications, and related fields. Fifth Edition. USA: Lippincott Williams \& Wilkins, 2005: 167-192.

27. Asociación Médica Mundial. Declaración de Helsinki de La Asociación Médica Mundial. Principios éticos para las investigaciones médicas en seres humanos. Seúl, Corea: Asociación Médica Mundial; 2008. Report No.: 59.

28. Gomez L, Vidal B, Morales L, et al. Low frequency repetitive transcranial magnetic stimulation in children with attention deficit/hyperactivity disorder. Preliminary results. Brain Stimul. 2014 Sep;7:760-762.

29. Rossi S, Hallett M, Rossini PM, Pascual-Leone A. Safety, ethical considerations, and application guidelines for the use of transcranial magnetic stimulation in clinical practice and research. Clin Neurophysiol. 2009 Dec;120:2008-2039.

30. Anderson B, Mishory A, Nahas Z, et al. Tolerability and safety of high daily doses of repetitive transcranial magnetic stimulation in healthy young men. J ECT. 2006 Mar;22:49-53.

31. Hadley D, Anderson BS, Borckardt JJ, et al. Safety, tolerability, and effectiveness of high doses of adjunctive daily left prefrontal repetitive transcranial magnetic stimulation for treatment-resistant depression in a clinical setting. J ECT. 2011 Mar;27:18-25.

32. Misra UK, Kalita J, Bhoi SK. High-rate repetitive transcranial magnetic stimulation in migraine prophylaxis: a randomized, placebo-controlled study. J Neurol. 2013 Nov;260:2793-2801.

33. Enticott PG, Fitzgibbon BM, Kennedy HA, et al. A double-blind, randomized trial of deep repetitive transcranial magnetic stimulation (rTMS) for autism spectrum disorder. Brain Stimul. 2014 Mar;7:206-211.

34. Amatachaya A, Auvichayapat N, Patjanasoontorn N, et al. Effect of anodal transcranial direct current stimulation on autism: a randomized double-blind crossover trial. Behav Neurol. 2014;2014:173073. doi: 10.1155/2014/173073. Epub;\%2014 Oct 30.:173073.

35. Lefaucheur JP, Andre-Obadia N, Poulet E, et al. [French guidelines on the use of repetitive transcranial magnetic stimulation (rTMS): safety and therapeutic indications]. Neurophysiol Clin. 2011 Dec;41:221-295.

36. Butler AJ, Shuster M, O'Hara E, Hurley K, Middlebrooks D, Guilkey K. A meta-analysis of the efficacy of anodal transcranial direct current stimulation for upper limb motor recovery in stroke survivors. J Hand Ther. 2013 Apr;26:162-170.

37. Nizard J, Lefaucheur JP, Helbert M, de CE, Nguyen JP. Non-invasive stimulation therapies for the treatment of refractory pain. Discov Med. 2012 Jul;14:21-31.

38. D'Urso G, Bruzzese D, Ferrucci R, et al. Transcranial direct current stimulation for hyperactivity and noncompliance in autistic disorder. World J Biol Psychiatry. 2015;16:361-366.

39. Casanova MF, Sokhadze E, Opris I, Wang Y, Li X. Autism spectrum disorders: linking neuropathological findings to treatment with transcranial magnetic stimulation. Acta Paediatr. 2015 Apr;104:346-355.

40. Datko M, Gougelet R, Huang MX, Pineda JA. Resting State Functional Connectivity MRI among Spectral MEG Current Sources in Children on the Autism Spectrum. Front Neurosci. 2016 Jun 9;10:258. doi: 10.3389/fnins.2016.00258. eCollection;\%2016.:258.

41. Takesaki N, Kikuchi M, Yoshimura Y, et al. The Contribution of Increased Gamma Band Connectivity to Visual Non-Verbal Reasoning in Autistic Children: A MEG Study. PLoS One. 2016 Sep 15;11:e0163133. 



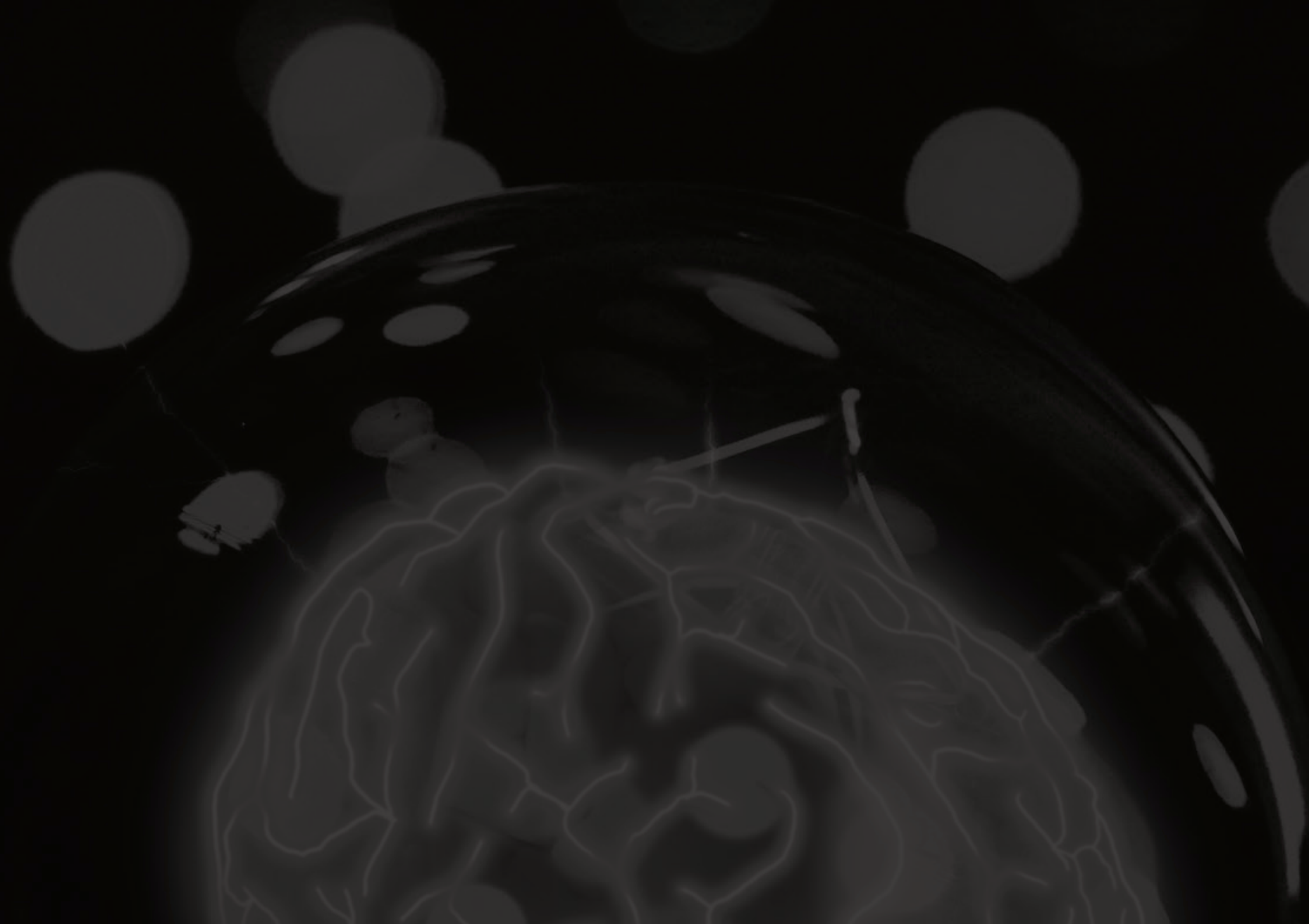

Revista Mexicana de Neurociencia, 2017; 18(3): 8-20 www.revmexneuroci.com

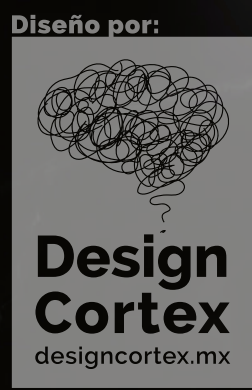

